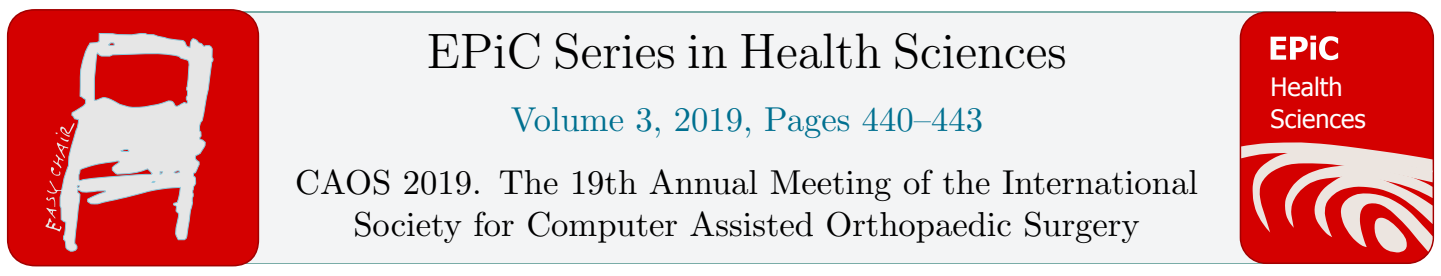

\title{
Does the Use of a CT Based 3D Plan Improve Joint Balancing in Total Knee Arthroplasty? A Multi-Center Study
}

\author{
Jingwei Zhang ${ }^{1}$, Manoshi Bhowmik-Stoker ${ }^{1}$, Laura Y. Scholl ${ }^{1}$, Caitlin \\ Condrey $^{2}$, Kirby D Hitt ${ }^{3}$, Kevin Marchand ${ }^{2}$, Robert Marchand ${ }^{2}$ \\ ${ }^{1}$ Stryker, Mahwah, NJ, USA \\ ${ }^{2}$ South County Hospital, Wakefield, RI, USA \\ ${ }^{3}$ Scott \& White Roney Bone \& Joint Institute, Temple, TX, USA \\ jingwei.zhangl@stryker.com, manoshi.bhowmik-stoker@stryker.com \\ laura.schollestryker.com, ccondreyescortho.com, \\ Kirby.Hitt@BSWHealth.org, kevin.marchand@me.com \\ marchand33@cox. net
}

\begin{abstract}
Studies have shown that dissatisfaction following TKA may stem from poor component placement and iatrogenic factors related to variability in surgical execution. A CT-based robotic assisted system allows surgeons to dynamically balance the joint prior to bone resection. This study aimed to determine if this system could improve TKA planning, reduce soft tissue releases, minimize bone resection, and accurately predict component size.

Six hundred and sixty-six cases undergoing primary robotic assisted TKA we enrolled in a prospective, multicenter study. Seven surgeons participated from seven US centers. Patient demographics and intraoperative surgical details were collected. Initial and final 3-dimensional alignment, component position, bone resection depths, use of soft tissue releases, knee balancing gaps, and component size were collected intraoperatively. Descriptive statistics were applied to determine the changes in these parameters between initial and final values.

In this study, 513 varus knees, 86 valgus knees, and 26 neutral knees were captured and stratified for analysis. Native deformity ranged from 12 degrees of valgus to 19 degrees of varus. $85 \%$ of all patients in this study did not require a soft tissue release. Complex deformities who required a soft tissue release were corrected on average to 3.36 degrees while cases without releases were corrected to 1.1 degree on average. All surgeons achieved their planned sizes on the tibia and femur more than $97.5 \%$ of the time within one size, and $100 \%$ of the time within two sizes. Flexion and extension gaps during knee balancing were within $2 \mathrm{~mm}$ (mean $1 \mathrm{~mm}$ ) for all knees.

New tools may allow for enhanced execution and predictable balance for TKA, which may improve patient outcomes. In this study, preoperative planning via CT scan allowed surgeons
\end{abstract}


to assess bony deformities and subtly adjust component position to reduce soft tissue trauma. Patient follow up is needed to determine clinical outcomes.

\section{Introduction}

Studies have shown that poor survivorships and clinical outcomes following TKA may stem from poor component placement, overall limb alignment, and iatrogenic factors related to variability in surgical execution [1,2]. A CT-based robotic-arm assisted system allows surgeons to dynamically balance the joint prior to bone resection and demonstrates great accuracy to plan (within 1 degree) [3]. This study aimed to determine if this system could improve TKA planning, reduce soft tissue releases, minimize bone resection, and accurately predict component size.

\section{Materials and Methods}

Six hundred and sixty-six cases undergoing primary robotic assisted TKA were enrolled in a prospective, multicenter study. Seven surgeons participated from seven US centers. Patient demographics and intraoperative surgical details were collected. Initial and final 3-dimensional alignment, component position, bone resection depths, use of soft tissue releases, knee balancing gaps, and component size were collected intraoperatively. Descriptive statistics were applied to determine the changes in these parameters between initial and final values.

\section{Results}

In this study, 513 varus knees, 86 valgus knees, and 26 neutral knees were captured and stratified for analysis. Native deformity ranged from 12 degrees of valgus to 19 degrees of varus. $85 \%$ of patients in this study did not require a soft tissue release regardless of their level of coronal or sagittal deformity (Figure 1). Complex deformities who required a soft tissue release were corrected on average to 3.36 degrees while cases without releases were corrected to 1.1 degree on average with the overall goal as traditional mechanical alignment (Figure 2). All surgeons achieved their planned sizes on the tibia and femur more than $97.5 \%$ of the time within one size, and $100 \%$ of the time within two sizes. All cases were adjusted intraoperatively prior to bony resection, resulting in an average change of $0.34 \mathrm{~mm}$ between initial and final cuts in all knees. Flexion and extension gaps during knee balancing were within $2 \mathrm{~mm}$ (mean $1 \mathrm{~mm}$ ) for all knees.

\section{Discussion and Conclusion}

In this study, preoperative planning via $\mathrm{CT}$ scan allowed surgeons to assess bony deformities and subtly adjust component position to reduce soft tissue trauma. only $15 \%$ of patients required soft tissue releases to correct the deformity to neutral. Component size predictions are relatively accurate within 1 size. RA technology allows surgeons to plan the case and make intraoperative component adjustments prior to bone cuts [4]. In literature, RA technology has been shown to reduce in post-operative pain and opioid usage which provides benefits to the patient and the hospital system [5]. Patient follow up is needed to determine clinical outcomes. 


\section{Figures}

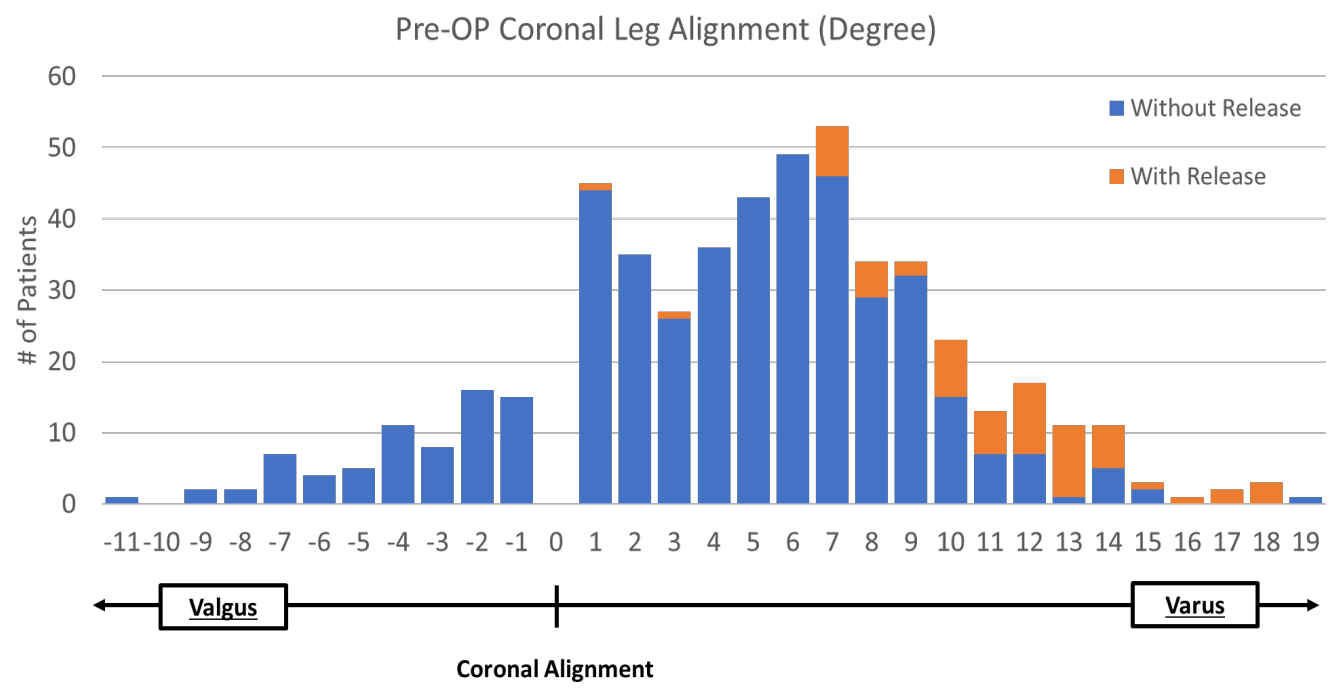

Figure 1. Patients with greater non-correctable varus (positive angles) deformities pre-operatively required a greater number of soft tissue releases. Valgus (negative angles) cases were corrected with bone cuts alone.

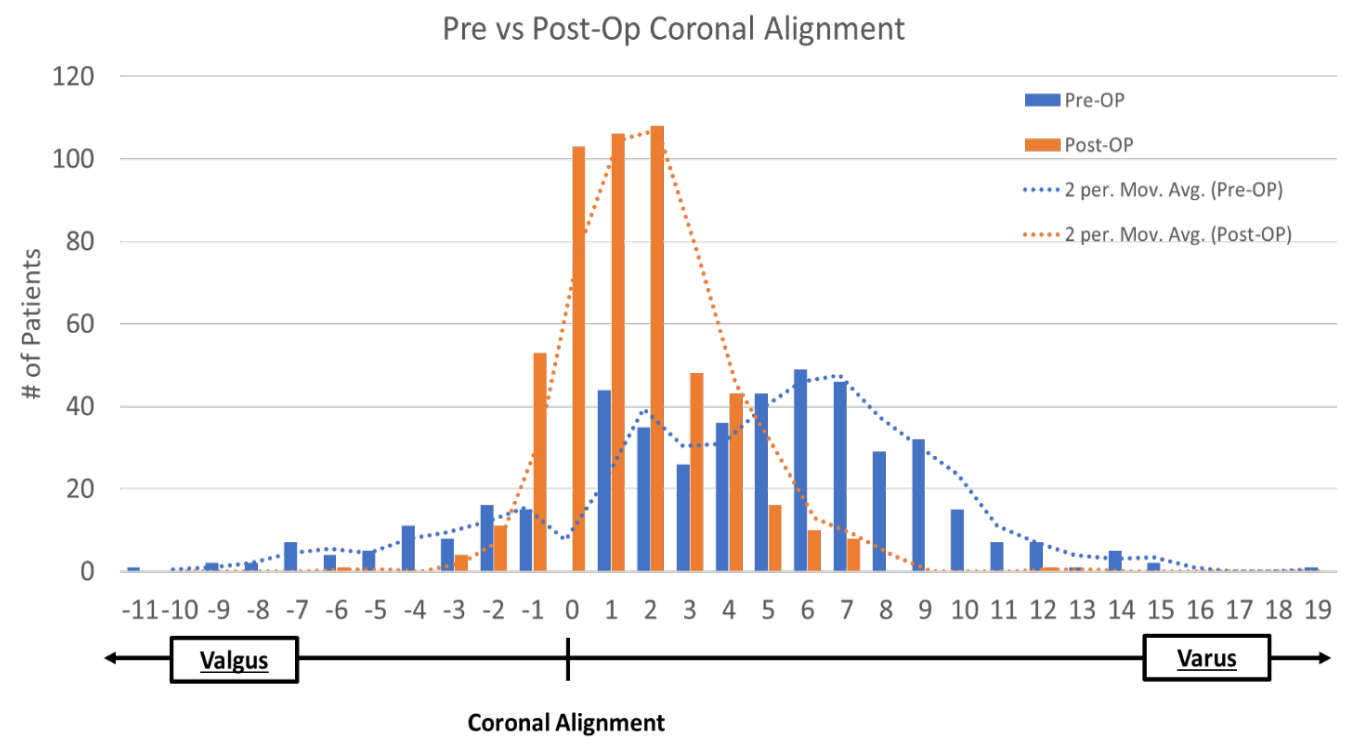

Figure 2. Pre-operative and post-operative coronal alignment following robotic assisted TKA. 


\section{References}

[1] Jeffery RS, Morris RW, Denham RA. Coronal alignment after total knee replacement. J Bone Joint Surg Br 1991;73:709.

[2] Huang NF1, Dowsey MM, Ee E, Stoney JD, Babazadeh S, Choong PF., Coronal alignment correlates with outcome after total knee arthroplasty: five-year follow-up of a randomized controlled trial, J Arthroplasty. 2012 Oct;27(9):1737-41. doi: 10.1016/j.arth.2012.03.058. Epub 2012 Aug 3.

[3] Hampp EL, Chughtai M, Scholl LY, Sodhi N, Bhowmik-Stoker M, Jacofsky DJ, Mont MA., Robotic-Arm Assisted Total Knee Arthroplasty Demonstrated Greater Accuracy and Precision to Plan Compared with Manual Techniques., J Knee Surg. 2018 May 1. doi: 10.1055/s-0038-1641729

[4] Marchand RC, Sodhi N, Bhowmik-Stoker M, Scholl L, Condrey C, Khlopas A, Sultan AA, Newman JM, Mont MA., Does the Robotic Arm and Preoperative CT Planning Help with 3D Intraoperative Total Knee Arthroplasty Planning?, J Knee Surg. 2018 Aug 15.

[5] Kayani B, Konan S, Tahmassebi J, Pietrzak JRT, Haddad FS., Robotic-arm assisted total knee arthroplasty is associated with improved early functional recovery and reduced time to hospital discharge compared with conventional jig-based total knee arthroplasty., Bone Joint J. 2018 Jul;100B(7):930-937. 